\title{
HIV seropositivity in patients with first-episode psychosis
}

S Mashaphu, MB ChB, FCPsych (SA)

D L Mkize, MB ChB, DCH (SA), MFGP (SA), DFM (SA), MMed (Psych)

Department of Psychiatry, Nelson Mandela School of Medicine, University of KwaZulu-Natal, Durban

Background. In order to gauge the impact of the HIV epidemic on psychotic disorders, the magnitude and causal direction of the association between HIV infection and psychosis need to be examined closely.

Objective. To determine the HIV seropositivity rate among adult patients presenting with first-episode psychosis (FEP) to Town Hill Hospital in Pietermaritzburg, KwaZulu-Natal.

Design. A cross-sectional, point-prevalence study was done over a b-month period.

Results. Of the 63 FEP patients in the study, 48 tested HIVnegative and 15 tested positive, giving a seroprevalence rate of $23.8 \%$.

Conclusion. The prevalence of HIV seropositivity is high among patients with FEP.

South Africa is currently experiencing one of the most severe HIV epidemics in the world.' By the end of 2005 there were 5.5 million people living with HIV in South Africa. ${ }^{2}$ Current population studies' estimate that HIV has infected $23 \%$ of the South African population, and that $13 \%$ of all people worldwide living with HIV live in South Africa. The levels of HIV infection tend to vary across different geographical regions in South Africa. Provincial data show that HIV incidence is higher in the northern and eastern provinces, and lower in the southern and western provinces. HIV incidence is also lower in the deep rural Northern Cape (12\%) and Eastern Cape (16\%). Of the 9 provinces, KwaZulu-Natal has the highest prevalence rate. ${ }^{2}$ Recent statistics indicate a $39.1 \%$ prevalence rate among antenatal clinic attendees in KwaZuluNatal, compared with $15.7 \%$ in the Western Cape. The reasons for these variations are not clear. ${ }^{2}$

An unpublished study on the seroprevalence of HIV infection and associated psychiatric syndromes in certified adult black male patients at Town Hill and Fort Napier hospitals done in 1992 revealed a seroprevalence rate of 2\% (S D Chetty - unpublished data, 1992). A subsequent study on the prevalence of HIV infection among admissions to Town Hill Hospital in 2003 revealed a prevalence rate of 26.5\% (K Mestry - unpublished data). Based on the above results and the escalating spread of HIV infection, one would assume that there would be high prevalence rates of HIV infection in patients presenting with firstepisode psychosis (FEP).

\section{HIV and psychosis}

Psychiatric symptoms and disorders are becoming increasingly evident in HIV-infected patients. ${ }^{3}$ The presence of psychotic symptoms during the course of HIV infection constitutes a wellknown but relatively rare complication. ${ }^{3} \mathrm{HIV}$-positive patients may exhibit symptoms of psychosis that are indistinguishable from those of primary functional disorders. ${ }^{4}$ Many cases arise in patients who already show physical symptoms of AIDS, but even then, the psychoses may be the first manifestation of HIV infection.

\section{HIV and FEP}

Psychotic symptoms may occur at any stage in the course of this illness, but they are usually late complications of HIV infection. ${ }^{6}$ FEP occurring in an HIV-infected patient poses a diagnostic dilemma, as one has to decide whether the presentation is organic or functional. This is further complicated by the fact that the age range of the peak incidence of HIV infection mirrors that for schizophrenia and mood disorders in the general population. ' It may therefore be difficult to establish a causal relationship between HIV infection and psychiatric morbidity. Some researchers argue that new-onset psychosis may be, at least in part, a manifestation of an HIV-associated encephalopathy. ${ }^{6}$ The aetiological association of the HIV infection remains to be established. ${ }^{8}$

\section{Objective}

The aim of the study was to determine HIV seropositivity among adult patients with FEP admitted to Town Hill Hospital in Pietermaritzburg, KwaZulu-Natal.

\section{Ethical consideration}

This research project was approved by the University of KwaZuluNatal Ethics Committee together with the Provincial Department of Health and Town Hill Hospital Ethics Committee. 


\section{Instruments}

The following instruments were used:

- A screening document that was used to screen all FEP patients presenting to Town Hill Hospital for suitability to participate in the research project. The instrument was used to determine the patient's past history, duration of illness, and medical and substance history.

- An information leaflet written in English and IsiZulu.

- A consent form.

- A demographic questionnaire.

\section{Methods}

A cross-sectional, point-prevalence study was undertaken. The study population comprised patients with FEP admitted to Town Hill Hospital. Town Hill is the largest psychiatric hospital in KwaZulu-Natal. It is a major referral centre for the surrounding regional and district hospitals. The study was conducted over a 6-month period ( 1 September 2005 - 28 February 2006).

The researcher (the principal investigator) requested the assistance of the attending psychiatrists to identify all new admissions with FEP (N=378). The final diagnosis was made by the multidisciplinary team based on Diagnostic and Statistical Manual of Mental Disorders (DSM) IV criteria. The patients were managed according to the standard guidelines until they were free of psychotic symptoms and ready to give consent. They were approached individually by the researcher, and written consent was obtained. All participants received pre-test counselling. The blood samples were collected by the researcher and sent to a private laboratory for HIV testing. The results were made available within 48 hours to all patients wanting to know their status. Posttest counselling was provided. Data were analysed using the statistical packages SSPS version 11.5 and Epi-Info version 6.04. Certain data were collapsed into broader categories to facilitate analysis. All primary psychotic disorders were collapsed into one category, viz. functional psychosis. Marital status was divided into two categories, i.e. 'single' and 'other'. The category 'other' included married, widowed and cohabiting individuals.

\section{Results}

Of 378 patients, 63 fulfilled all the inclusion criteria. There were 13 females and 50 males, reflecting the demographic admission patterns at Town Hill Hospital, i.e. more males than females. The demographic characteristics are given in Table I. The remaining group of patients did not participate in the research project for a variety of reasons. Some were lost to follow-up or had a coexisting medical condition; others refused to participate in the study or were deemed not capable of giving informed consent. The majority of female patients were married, widowed or cohabiting, while the majority of the males were single. Fifteen patients tested seropositive (6 females and 9 males), and 48 were negative. Of those who tested positive, 10 were blacks, 3 were coloured and 2 were Indian. The majority of the patients who tested positive lived in urban areas, were unemployed, and had secondary education (Table I).

The 15 patients who were HIV-positive had the following diagnoses: schizophreniform disorder (N=7), substance-induced psychotic disorder $(N=3)$, schizophrenia $(N=1)$, bipolar mood disorder $(N=1)$, brief psychotic disorder $(N=1)$, dementia ( $N$ $=1$ ), and major depressive episode with psychotic features $(N$ $=11$.

The average duration of symptoms before admission was 14 weeks.

\section{Discussion}

The study found a high prevalence of HIV seropositivity (23.8\%) among patients with FEP. This represents a significant rise in the HIV seroprevalence rates among psychiatric patients admitted to Town Hill Hospital. A previous study found a lower prevalence rate of 2\% in 1992 (S D Chetty - unpublished data). Rates in other studies done on new-onset psychosis in patients with

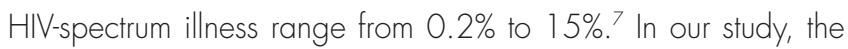
majority of the females were married, cohabiting or widowed. It can be reasonably assumed that these individuals had had a regular sexual partner at some stage in their lives. None of the patients had a history of substance use. This was an important factor because substance use is usually related to an increase in risk-taking behaviour and sexual indiscretions. Despite HIV/ AIDS education, substance users continue to engage in HIV risk behaviours. Those who inject drugs, particularly opioids and cocaine, may share or fail to clean injection equipment and often engage in unsafe sex. ${ }^{9}$ A study by Susser et al. ${ }^{10}$ in 1995 on the sexual behaviour of homeless mentally ill men found an HIV prevalence rate of $19 \%$. The majority of these individuals had sex occasionally or not at all, but many of them had unprotected sex with non-monogamous partners. Added to this, they had comorbid cocaine abuse or dependence that put them at high risk for contracting the virus.

The average duration of psychotic symptoms at the time of recruitment was 14 weeks. It can reasonably be assumed that 
Table I. Demographic characteristics and HIV status of the sample $(N(\%))$

\begin{tabular}{|c|c|c|c|c|}
\hline & HIV-positive ( $N=15)$ & HIV-negative $(N=48)$ & Total $(N=63)$ & p-value \\
\hline Gender & & & & $0.034^{*}$ \\
\hline Male & $9(18.0)$ & $41(82.0)$ & $50(100)$ & \\
\hline Female & $6(46.2)$ & 7 (53.8) & $13(100)$ & \\
\hline Residence & & & & 0.214 \\
\hline Rural & $6(17.6)$ & $28(82.4)$ & $34(100)$ & \\
\hline Urban & $9(31.0)$ & $20(69.0)$ & $29(100)$ & \\
\hline Employment & & & & 0.758 \\
\hline Employed & $2(20.0)$ & $8(80.0)$ & $10(100)$ & \\
\hline Unemployed & $13(24.5)$ & $40(75.5)$ & $53(100)$ & \\
\hline Education & & & & 0.479 \\
\hline No education & $0(0)$ & $1(100)$ & $1(100)$ & \\
\hline Secondary & $13(29.5)$ & $31(70.5)$ & $44(100)$ & \\
\hline Tertiary & 0 & $6(100)$ & $6(100)$ & \\
\hline Marital status & & & & 0.034 \\
\hline Single & $9(18.0)$ & $41(82.0)$ & $50(100)$ & \\
\hline Other & $6(46.2)$ & $7(53.8)$ & $13(100)$ & \\
\hline
\end{tabular}

the HIV infection predated the onset of the psychotic symptoms. The majority of patients were diagnosed with schizophreniform disorder. This was an unexpected finding since the researcher had anticipated higher prevalence rates in bipolar patients, due to high risk-taking behaviour because of impaired judgement in this group. There was no conclusive evidence that the HIV itself caused the psychosis, but this aetiology cannot be ruled out. New-onset psychosis may be, at least in part, a manifestation of an HIV-associated encephalopathy. ${ }^{5}$ To support this further, none of the participants had a known co-morbid medical condition that could account for the current psychiatric presentation. This finding suggests that the symptoms of psychosis in those infected with HIV are not always related to systemic and cerebral complications of HIV.

\section{Limitations of the study}

The main limitation of this study was the sample size. This was due to a variety of reasons: (i) the number of new admissions to Town Hill Hospital dropped significantly after the New Mental Health Act was promulgated; (ii) the pre- and post-test counselling process was time-consuming, and as a result a high number of patients were lost to the study group during their hospital stay; (iii) the recruitment period of 6 months was too short; and (iv) there was no demographic representation for the white population.

Based on all the above limitations, the results of this study cannot be generalised to the general population. Further research needs to be done to explore the aetiological relationship between FEP and HIV seropositivity.

\section{Conclusion and recommendations}

This study demonstrated a high prevalence of HIV seropositivity among patients with FEP. This prevalence is in keeping with prevalence figures in South Africa and the rest of the world. ${ }^{4}$ Estimates of the frequency of HIV infection in patients with preexisting mental illness range between $0.2 \%$ and $7 \%$. Estimates of new-onset psychosis in patients with HIV-spectrum illness range between $0.2 \%$ and $15 \%$ and may increase as the stage of HIV infection progresses. Regardless of which illness came first, their occurrence together appears to be associated with more morbidity and mortality than would be expected with either illness alone. The high prevalence rate suggests a possible aetiological association between HIV infection and FEP. However, the aetiological link remains to be established.

Based on the above findings, it is highly recommended that HIV testing be considered in patients presenting with FEP. Similarly, it is also reasonable to consider co-morbid HIV infection in psychotic patients. Psychiatrists are in a better position to actively educate their patients on prevention of HIV infection. " The need for implementation of risk-reduction programmes in psychiatric facilities has to be emphasised because a significant proportion 
of patients could be infected with HIV. Particular emphasis should be placed on voluntary counselling and testing (VCT). Further research is recommended on: (i) the prevalence of HIV seropositivity in a large sample of FEP patients; and (ii) the aetiological relationship between HIV seropositivity and FEP.

\section{References}

1. UNAIDS, December 1999. http:www.africafocus.org/doc/06/aids0602.php (last accessed 30 March 2006).

2. Gilbert L, Walker L. HIV and AIDS in South Africa. Cad Saude Publica May/June 2002; 18 (3): 651-660.

3. Susser E. HIV infection among young adults with psychotic disorders. Am J Psychiatry 1997; 154: 864-866.
4. Grant I, Atkinson JH. Neuropsychiatric aspects of HIV infection and AIDS. In: Kaplan HI, Saddock BJ, eds. Comprehensive Textbook of Psychiatry. Vol. 1. 7th ed. Baltimore: Williams \& Wilkins, 1999

5. Sewell DD, Jeste DV, Atkinson JH, et al. HIV associated psychosis. Am J Psychiatry 1994; 151: 237-242.

6. Doyle ME, Labbate LA. Incidence of HIV infection among patients with new-onset psychosis. Psychiatric Services 1997; 48: 237-238

7. Sewell DD. Schizophrenia and HIV. Schizophr Bull 1996; 22: 465-473

8. Harris MJ, Jeste DV, Gleghorn A, Sewell DD. New-onset psychosis in HIV-infected patients. J Clinical Psychiatry 1992; 53; 293-294.

9. Des Jarlais DC, Friedman SR, Woods J, Milliken J. HIV infection among intravenous drug users. In: Comprehensive Textbook of Substance Abuse. 2nd ed. Baltimore: Williams \& Wilkins, 1992: 734-743

10. Susser $E$, Valentia $E$, Miller $M$, et al. Sexual behaviour of homeless mentally ill men at risk for HIV. Am J Psychiatry 1995; 152: 583-587.

1 1. Chung JY, Suarez AP, Zarin DA, Pincus HA. Psychiatric patients and HIV. Psychiatr Serv 1999: 50 (April): 487 